\title{
BMJ Open The association between objectively measured sitting and standing with body composition: a pilot study using MRI
}

\author{
L Smith, ${ }^{1}$ E L Thomas, ${ }^{2} \mathrm{~J}$ D Bell, ${ }^{2}$ M Hamer ${ }^{1}$
}

To cite: Smith L, Thomas EL, Bell JD, et al. The association between objectively measured sitting and standing with body composition: a pilot study using MRI. BMJ Open 2014;4:e005476. doi:10.1136/bmjopen-2014005476

- Prepublication history and additional material is available. To view visit the journal (http://dx.doi.org/10. 1136/bmjopen-2014005476).

Received 14 April 2014 Revised 23 May 2014 Accepted 27 May 2014

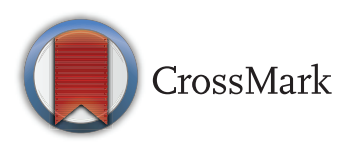

${ }^{1}$ Department of Epidemiology and Public Health, University College London, London, UK ${ }^{2}$ Metabolic and Molecular Imaging Group, MRC Clinical Sciences Centre, Imperial College London, London, UK

Correspondence to Dr L Smith; lee.smith@ucl.ac.uk

\section{ABSTRACT}

Objective: To investigate the association between objectively measured sitting and standing, using a postural allocation technique, with MRI-assessed body composition.

Design: The present study was a cross-sectional pilot study.

Setting: Participants were examined at one centre located in London, UK.

Participants: Normal weight Caucasian women (30.9 \pm 6.1 years; body mass index (BMI), $22.9 \pm 3.4 \mathrm{~kg} / \mathrm{m}^{2}$ ) with desk-bound occupations were recruited to minimise variability in body composition outcomes. A convenience sample of 12 women was recruited in January 2014 from University College London.

Outcome measures: For each participant a number of body composition variables were attained from a single whole-body MRI session. Main outcome variables included: total and liver adiposity, visceral/ subcutaneous fat ratio and BMI. Main exposure variables included: average sitting time, standing:sitting ratio and step count. Pearson correlations were carried out to examine associations between different activity categories and body composition variables.

Results: There were significant correlations between average daily sitting and liver adiposity and visceral/ subcutaneous abdominal fat ratio $(r=0.66$ and 0.64 , respectively); standing:sitting ratio was moderately correlated with liver adiposity and visceral/ subcutaneous abdominal fat ratio $(r=-0.53$ and -0.45 ); average daily step count was moderately correlated with liver adiposity, total adiposity and visceral/subcutaneous abdominal fat ratio $(r=-0.45$, -0.46 and -0.51 , respectively).

Conclusions: This pilot study has provided preliminary evidence of relationships between objectively measured sitting and standing and precise measures of body composition.

\section{INTRODUCTION}

There is a growing body of literature that suggests sedentary behaviours-defined as any waking behaviour characterised by energy

\section{Strengths and limitations of this study}

- This is the first study to show an association between objectively measured sitting and standing, using a postural allocation technique, and MRI-assessed body composition.

- The data collection protocol and tools used within this pilot study are feasible and can be implemented into subsequent experimental trials.

- It was not feasible to make multiple statistical adjustments in our analyses owing to the small sample size.

expenditure below 1.5 metabolic equivalents while in a sitting or reclined posture-are associated with higher risk of cardiovascular disease (CVD) and mortality, after statistical adjustment for moderate-to-vigorous-intensity physical activities (MVPA; eg, brisk walking). ${ }^{1}$ This has large public health relevance in light of objective data from general adult population studies in the USA and Great Britain that show on average adults spend approximately $60-70 \%$ of their waking hours in sedentary behaviours. $^{2}$ Indeed, westernised society is geared towards promoting sedentary lifestyles (ie, screen-based entertainment, motorised transport, etc), thus, developing strategies to combat sedentary behaviour is crucial.

Such a strategy might not necessarily involve exercise of moderate or vigorous intensity, as interventions to increase exercise levels have proved challenging. ${ }^{3}{ }^{4}$ Instead, given the barriers to structured exercise (eg, motivation, cost, access and time, etc), we might consider more subtle lifestyle approaches that are primarily designed to displace sedentary behaviour (ie, sitting) with forms of lighter intensity (incidental) activity (eg, standing). If lifestyle population-activity patterns can be shifted from predominantly sedentary to the next lowest physical activity (PA) category (standing), this may have 
public health benefit given the low proportion of individuals meeting current PA guidelines.

Until now, limited epidemiological evidence has been generated on the associations between light-intensity activity and health. This is partially owing to measurement issues; self-reported PA questionnaires are designed to capture MVPA and there are technical limitations in differentiating between sitting, standing and other forms of light activity when interpreting objective activity data. The most commonly used accelerometer, the Actigraph, quantifies time spent in different intensities of activity by summing time above and below specified count thresholds. This method works reasonably well for identifying MVPA, but is less accurate for distinguishing between sedentary and light activity (ie, between sitting and standing). ${ }^{5}$ Thus, methods that employ postural allocation may be more reliable, which have only recently become readily available.

Some experimental evidence is beginning to emerge in this area. For example, one study manipulated sitting time and PA over 1 day under free-living conditions. The results indicated that replacing sitting with longer periods of light activity was more beneficial for metabolic health than $1 \mathrm{~h}$ of vigorous exercise despite equivalent daily energy expenditure in each treatment group. ${ }^{6}$ In a laboratory-controlled trial conducted over an $8 \mathrm{~h}$ period, interrupting sitting time every 20 min with short $2 \mathrm{~min}$ bouts of light-intensity or moderate-intensity walking was shown to lower postprandial glucose and insulin levels in overweight/obese adults. ${ }^{7}$ In another study, using continuously monitored capillary blood glucose, there was a $43 \%$ reduction in blood glucose excursion during an afternoon (185 min) of standing compared with sitting in desk-based workers. ${ }^{8}$ In a pilot study replacing sitting workstations with sit-stand workstations, employees reduced sitting time by $137 \mathrm{~min} /$ day and increases in high-density lipoprotein cholesterol were observed at 3 months follow-up. ${ }^{9}$ However, the biological mechanisms underlying these effects still remain unclear, although increased muscle activation during standing could be an important underlying mechanism, for example, by increasing skeletal muscle metabolism. Replacing a sitting workstation with a standing workstation was shown to increase daily energy expenditure, ${ }^{8}$ thus the longer term benefits might also include reductions in total, visceral and liver adiposity. A reduction in total and visceral adiposity is known to have a favourable impact on a range of CVD risk factors including inflammatory markers, lipids and glycemic control. ${ }^{10}$ Liver adiposity is of particular interest as it has been linked to metabolic risk and worsening insulin resistance. ${ }^{11}$ Several exercise training studies have demonstrated reductions in visceral adiposity in the absence of weight loss. ${ }^{12}$ However, the relationship between light PA (standing) and total, visceral and liver adiposity has yet to be investigated using precise imaging techniques.

Further research is needed to aid in the understanding of the relationships between objectively measuring sitting and standing, using an objective postural allocation technique, and measures of total, visceral and liver adiposity, using precise imaging techniques. This will contribute to the small but growing body of literature that aims to inform policy and intervention on the health benefits of displacing sitting with standing.

\section{AIM}

In the present study, several contemporary methods were used, including an objective postural allocation technique in combination with MRI, to aid in the understanding of the relationships between sitting/standing and body composition. This data collection was primarily designed to inform a large experimental trial that will investigate the impact of displacing sitting with standing on total, visceral and liver adiposity. This pilot data will (1) inform the underlying rationale of the trial by producing evidence, if it exists, of relationships between objective measures of sitting and standing and body composition and (2) generate an effect size on which to base sample size calculations to inform the main trial.

\section{METHODS}

\section{Design, participants and sample size}

This cross-sectional pilot study was carried out in 12 healthy Caucasian women. The sample size for this pilot was based on previous published work, which has shown that significant differences in body composition could be readily observed in cross-sectional studies of 10 or less volunteers. ${ }^{13}$ Normal weight women with deskbound occupations were selected from a larger cohort to minimise variability in age, weight and overall anthropometry. A convenience sample was recruited in January 2014 from University College London. We randomly invited 12 women who met our criteria to take part in the study. All women invited agreed to take part. One week prior to data collection trained research staff met with the participants to administer the participant information sheets and explain the study protocol.

\section{Measures of adiposity}

For each participant, a range of body composition variables were attained from a single whole-body MRI session lasting approximately $45 \mathrm{~min}$. For the purpose of the present study, we defined our main outcomes as a priori, which included body mass index (BMI), total litres of body adiposity (L), liver adiposity (\%) and visceral/subcutaneous abdominal fat ratio. Whole-body MRI and liver adiposity (\%) were obtained on a $1.5 \mathrm{~T}$ Phillips Achieva scanner (Philips Medical Systems, Best, The Netherlands) as previously described. ${ }^{14}$ Each participant was asked not to participate in strenuous exercise or drink alcohol $24 \mathrm{~h}$ before their scan. Each participant was also requested not to eat and only to drink water from 20:00 on the evening before their scan until the scan was completed. Trained research staff 
measured participants' height and weight from which BMI was calculated in $\mathrm{kg} / \mathrm{m}^{2}$.

\section{Free-living activity}

Immediately after MRI, an ActivPal accelerometer/ inclinometer device (http://www.paltechnologies.com/) was attached to the participants' thigh mid-way between their right hip and knee and covered with waterproof Tegaderm dressing. The ActivPal classifies an individual's free-living activity into periods spent sitting, standing and walking, which it has been validated for. ${ }^{15}$ The ActivPal's inclinometer and its unique positioning on the thigh allows the device to distinguish between sitting and standing using proprietary algorithms, which previous objective PA monitors have been unable to do. The device was worn all day every day (participants were instructed to wear the device during sleep and bathing) for seven full consecutive days. Bespoke software provided by Paltech was used to categorise activity periods into sitting/lying, standing and stepping, in addition to providing average daily step count. The data are presented as average daily waking time in hours per day (classified as 7:00 to 23:59) spent sitting, standing and stepping.

Written informed consent was obtained from all participants.

\section{Analysis}

Characteristics of the study population, average daily time spent sitting, standing and walking and the main body composition outcomes were summarised using descriptive statistics. We performed Pearson correlations to examine associations between different activity categories and the body composition data. For illustrative purposes, we also created a standing:sitting ratio and derived two groups based on the median split (high and low). Independent $t$ tests were performed to compare the main body composition outcomes between groups of high and low standing:sitting ratio. We extracted the partial $\mathrm{R}^{2}$ statistic from the correlation between sitting time and liver adiposity to inform a power calculation to provide a sample size for the main trial.

\section{RESULTS}

Of the 12 women who took part, all provided valid MRI and ActivPal data. The volunteers had a mean age of $30.9 \pm 6.1$ years, a mean BMI of $22.9 \pm 3.4$ and achieved an average of $9993 \pm 5146$ steps a day (table 1 contains all descriptive statistics for the study sample). On average, participants spent $12.7 \pm 1.3 \mathrm{~h}$ a day sitting, $3.2 \pm 0.9 \mathrm{~h}$ a day standing, $1.8 \pm 0.8 \mathrm{~h}$ a day stepping and the remainder in sleep.

There were significant correlations between average daily sitting and liver adiposity and visceral/subcutaneous abdominal fat ratio $(\mathrm{r}=0.66$ and 0.64 , respectively; see online supplementary file 1 for scatter plots between sitting and body composition outcomes); standing:sitting
Table 1 Descriptive statistics of study sample

\begin{tabular}{lcl}
\hline Variable & Mean/SD & Range \\
\hline Age & $30.9 \pm 6.1$ & $24-45$ \\
BMI $\left(\mathrm{kg} / \mathrm{m}^{2}\right)$ & $22.9 \pm 3.4$ & $18.1-28.1$ \\
Total body adipose tissue (L) & $24.1 \pm 9.9$ & $13.2-44.4$ \\
Liver adiposity (\%) & $0.52 \pm 0.73$ & $0.12-2.56$ \\
Visceral/subcutaneous & $0.25 \pm 0.09$ & $0.13-0.38$ \\
abdominal fat ratio & & \\
Average sitting time (h/day) & $12.7 \pm 1.3$ & $11.0-15.0$ \\
Average standing time (h/day) & $3.2 \pm 0.9$ & $1.4-4.4$ \\
Average stepping (h/day) & $1.8 \pm 0.8$ & $0.6-3.1$ \\
Average daily step count & $9993 \pm 5146$ & $2918-19995$ \\
Average daily energy & $24.4 \pm 2.3$ & $22.6-30.2$ \\
expenditure (MET-h) & & \\
\hline n=12. & & \\
BMl, body mass index. & &
\end{tabular}

ratio was moderately correlated with liver adiposity and visceral/subcutaneous abdominal fat ratio $(r=-0.53$ and -0.45 ); average daily step count was moderately correlated with liver adiposity, total adiposity and visceral/subcutaneous abdominal fat ratio $(\mathrm{r}=-0.45,-0.46$ and -0.51 , respectively; table 2). Scatter plots of these associations are presented as supplementary material (see online supplementary figures S1-S4). We observed weak associations between all activity categories with BMI. Figure 1 presents comparisons between groups of high and low standing:sitting ratio in relation to the main body composition outcomes. A higher ratio of standing: sitting was consistently associated with lower levels of adiposity. Since the observed associations with sitting may have been influenced by vigorous exercise, we performed sensitivity analyses that removed two participants recording over 15000 steps (indicative of vigorous exercise). In these analyses $(n=10)$, the associations between sitting and adiposity remained largely unchanged; average daily sitting remained associated with liver adiposity $(\mathrm{r}=0.65, \mathrm{p}=0.043)$ and visceral/subcutaneous abdominal fat ratio $(\mathrm{r}=0.73, \mathrm{p}=0.017)$.

\section{Power calculation}

A power calculation was carried out in G-Power to provide a sample size for the main trial. The calculation

Table 2 Correlations between ActivPal and MRI measures

\begin{tabular}{|c|c|c|c|c|}
\hline & BMI & $\begin{array}{l}\text { Total } \\
\text { adiposity }\end{array}$ & Liver fat & $\begin{array}{l}\text { Visceral/ } \\
\text { subcutaneous } \\
\text { abdominal } \\
\text { fat ratio }\end{array}$ \\
\hline Sitting & -0.09 & 0.10 & $0.66^{*}$ & $0.64^{*}$ \\
\hline Stand:sit ratio & 0.24 & 0.08 & $-0.53 \dagger$ & -0.45 \\
\hline $\begin{array}{l}\text { Average step } \\
\text { count }\end{array}$ & -0.22 & -0.46 & -0.45 & $-0.51 \dagger$ \\
\hline
\end{tabular}


Figure 1 (A) Body mass index; (B), total adipose fat (L); (C) liver fat (\%); (D) visceral/subcutaneous abdominal fat ratio; $n=12$. The high:low cut point was $\geq 0.27$.
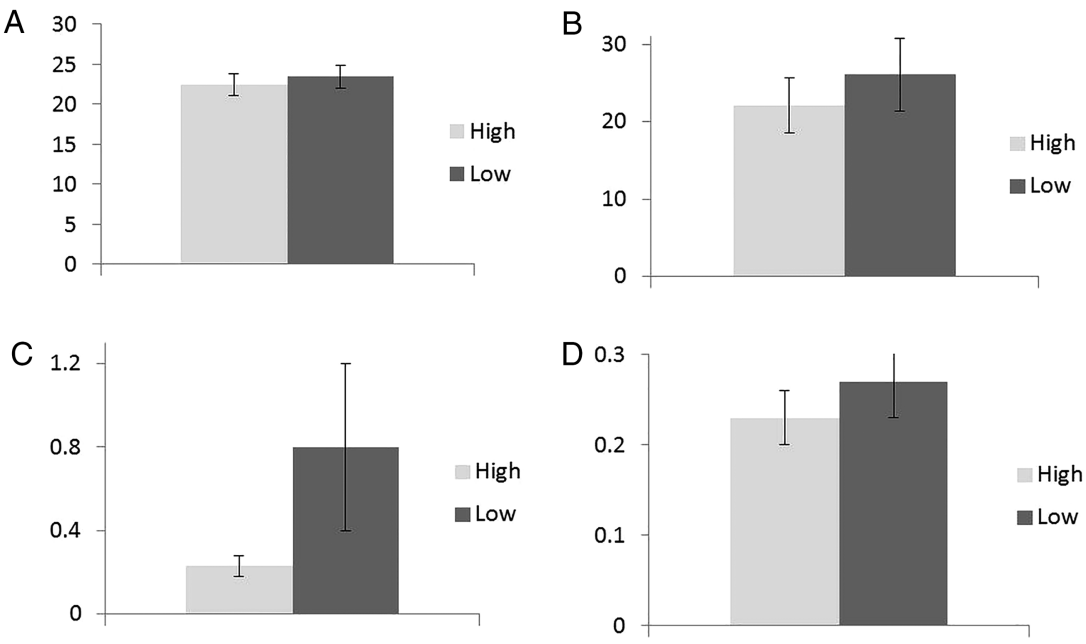

was based on the correlation between sitting time and liver fat: per $1 \mathrm{~h} /$ day sitting was associated with 0.48 (SE 0.17 ) unit increase in liver fat (partial $R^{2}=0.43$ ). In G-power this equates to an effect size $\mathrm{f}^{2}=0.75$, and suggests that a sample size of 20 per group would provide us with $95 \%$ power at $5 \%$ significance level (two-tailed) to detect differences.

\section{DISCUSSION}

The aim of the present study was to investigate the association between objectively measured sitting and standing, using a postural allocation technique (an accelerometer/inclinometer attached to the participant's thigh mid-way between the hip and the knee), with MRI-assessed body composition. Average daily sitting time was associated with liver adiposity and visceral/subcutaneous abdominal fat ratio. Previous studies have attempted to investigate these associations, but without the benefit of the existing gold standard techniques for body composition or full postural allocation measurements. In a recent study using CT, self-reported leisure-time sitting was associated with pericardial fat, but not with any other fat depots. ${ }^{16}$ We have previously reported associations between objectively assessed sedentary time (Actigraph) and pericardial fat although the relationship did not persist after adjusting for MVPA. ${ }^{17}$ Numerous studies have been carried out to investigate the relationship between sedentary time and BMI in adults and found mixed results. For example, one study carried out in a sample of 881 adults residing in Australia found no significant relationship between change in participant-reported TV viewing time and change in BMI, although a cross-sectional association was found between TV viewing time and BMI at baseline, in women only. ${ }^{18}$ In another study carried out in a sample of 3127 adults residing in southern France, participant-reported TV viewing time was associated with BMI in both sexes. ${ }^{19}$ In the Whitehall II prospective study, BMI predicted sitting time at follow-up but the converse was not found. ${ }^{20}$ Conflicting findings may be partially explained by the fact that BMI is a poor indicator of adiposity. Moreover, participants may not be able to recall $\mathrm{TV}$ viewing time accurately and $\mathrm{TV}$ viewing time may be a poor indicator of total sitting.

In comparison to previous research the present study used precise objective measures of sitting time and body composition. Interestingly, a higher ratio of standing: sitting was associated with lower levels of total and liver adiposity and visceral/subcutaneous fat ratio, providing preliminary cross-sectional evidence of the potential influence of light PA (standing) on body composition. These findings, although using a more proximal outcome, support previous literature that has found selfreported standing time is inversely related to CVD mortality in adults. ${ }^{21}$

The present pilot study found weak associations between all activity categories and BMI. BMI is a poor measure of adiposity in comparison to MRI since it cannot distinguish between visceral and subcutaneous fat depots. Since visceral and ectopic fat are thought to be more detrimental to health than subcutaneous, ${ }^{10}{ }^{11}$ it is important to distinguish between different types of fat. Furthermore, the numerator in the BMI calculation 'total body weight' does not distinguish between lean and fat mass. Therefore, an individual with high levels of lean mass may be classified as having a high BMI; whereas an individual who is of normal weight but has excess body fat may be classified as having a normal BMI. This might partly explain why several exercise training studies have demonstrated reductions in visceral adiposity in the absence of weight loss. ${ }^{12}$

The data collection protocol and tools used within this small pilot study are feasible and can be implemented into the subsequent experimental trial; a $100 \%$ response rate was achieved and no participant dropped out of the study. Moreover, all participants provided a full ActivPal dataset (seven complete days) and adhered to the wear protocol. However, it should be noted that the subsequent experimental trial will require two identical data 
collection sessions to assess the impact of displacing sitting with standing on body composition.

It was not feasible to make multiple statistical adjustments in our analyses owing to the small sample size, thus associations between sitting and adiposity may have been confounded by vigorous exercise. However, we selected a homogeneous sample and the removal of two highly active participants in our sample did not change the results. Given the cross-sectional nature of this pilot study the direction of the observed associations remains unknown. Moreover, the representativeness of the findings is limited owing to the small sample size of healthy Caucasian women residing in London. However, the aim of this pilot study was to provide novel data to support the underlying rationale and generate a sample size for a subsequent experimental trial.

Inclusion criteria for the experimental trial, that this pilot study was carried out to inform, will be overweight/obesity. We will use a number of biomedical outcomes in the main trial including body composition (MRI), and biochemical risk markers (lipids, inflammatory markers, glucose).

\section{CONCLUSION}

This pilot study has provided preliminary evidence of the strong relationships between objectively measured sitting and standing (an accelerometer/inclinometer attached to the participant's thigh mid-way between the hip and the knee) and precise measures of body composition.

Contributors LS and MH designed the study. All authors contributed to development of the study protocol. LS and MH drafted the manuscript. ELT and JDB assisted in drafting the manuscript. All authors read and approved the final manuscript.

Funding This research received no specific grant from any funding agency in the public, commercial or not-for-profit sectors.

Competing interests LS is supported by the National Institute for Health Research's School for Public Health Research. MH is supported by the British Heart Foundation (RE/10/005/28296).

Patient consent Obtained.

Ethics approval Hammersmith Research Ethics Committee (ref nos: 07/ Q0411/19 and 06/Q0411/173).

Provenance and peer review Not commissioned; externally peer reviewed.

Data sharing statement No additional data are available.

Open Access This is an Open Access article distributed in accordance with the Creative Commons Attribution Non Commercial (CC BY-NC 4.0) license, which permits others to distribute, remix, adapt, build upon this work noncommercially, and license their derivative works on different terms, provided the original work is properly cited and the use is non-commercial. See: http:// creativecommons.org/licenses/by-nc/4.0/

\section{REFERENCES}

1. Grøntved A, Hu FB. Television viewing and risk of type 2 diabetes, cardiovascular disease, and all-cause mortality: a meta-analysis. JAMA 2011;305:2448-55.

2. Stamatakis $\mathrm{E}$, Hamer M, Tilling $\mathrm{K}$, et al. Sedentary time in relation to cardio-metabolic risk factors: differential associations for self-report vs accelerometry in working age adults. Int $\mathrm{J}$ Epidemiol 2012;41:1328-37.

3. Kinmonth AL, Wareham NJ, Hardeman W, et al. Efficacy of a theory-based behavioural intervention to increase physical activity in an at-risk group in primary care (ProActive UK): a randomised trial. Lancet 2008;371:41-8.

4. Pavey TG, Taylor AH, Fox KR, et al. Effect of exercise referral schemes in primary care on physical activity and improving health outcomes: systematic review and meta-analysis. BMJ 2011;343: d6462.

5. Kozey-Keadle S, Libertine A, Lyden K, et al. Validation of wearable monitors for assessing sedentary behavior. Med Sci Sports Exerc 2011;43:1561-7.

6. Duvivier BM, Schaper NC, Bremers MA, et al. Minimal intensity physical activity (standing and walking) of longer duration improves insulin action and plasma lipids more than shorter periods of moderate to vigorous exercise (cycling) in sedentary subjects when energy expenditure is comparable. PLOS ONE 2013;8:e55542.

7. Dunstan DW, Kingwell BA, Larsen R, et al. Breaking up prolonged sitting reduces postprandial glucose and insulin responses. Diabetes Care 2012;35:976-83.

8. Buckley JP, Mellor DD, Morris M, et al. Standing-based office work shows encouraging signs of attenuating post-prandial glycaemic excursion. Occup Environ Med 2014;71:109-11.

9. Alkhajah TA, Reeves MM, Eakin EG, et al. Sit-stand workstations: a pilot intervention to reduce office sitting time. Am J Prev Med 2012:43:298-303

10. Pou KM, Massaro JM, Hoffmann U, et al. Visceral and subcutaneous adipose tissue volumes are cross-sectionally related to markers of inflammation and oxidative stress: the Framingham Heart Study. Circulation 2007;116:1234-41.

11. Koska J, Stefan N, Permana PA, et al. Increased fat accumulation in liver may link insulin resistance with subcutaneous abdominal adipocyte enlargement, visceral adiposity, and hypoadiponectinemia in obese individuals. Am J Clin Nutr 2008;87:295-302.

12. Lee S, Kuk JL, Davidson LE, et al. Exercise without weight loss is an effective strategy for obesity reduction in obese individuals with and without type 2 diabetes. J Appl Physiol 2005;99:1220-5.

13. Ostberg JE, Thomas EL, Hamilton G, et al. Excess visceral and hepatic adipose tissue in Turner syndrome determined by magnetic resonance imaging: estrogen deficiency associated with hepatic adipose content. J Clin Endocrinol Metab 2005;90:2631-5.

14. Thomas EL, Hamilton G, Patel N, et al. Hepatic triglyceride content and its relation to body adiposity: a magnetic resonance imaging and proton magnetic resonance spectroscopy study. Gut 2005;54:122-7

15. Grant M, Ryan C, Tigbe $\mathrm{W}$, et al. The validation of a novel activity monitor in the measurement of posture and motion during everyday activities. Br J Sports Med 2006;40:992-97.

16. Larsen BA, Allison MA, Kang E, et al. Associations of physical activity and sedentary behavior with regional fat deposition. Med Sci Sports Exerc 2014;46:520-8.

17. Hamer M, Venuraju SM, Urbanova L, et al. Physical activity, sedentary time, and pericardial fat in healthy older adults. Obesity (Silver Spring) 2012;20:2113-17.

18. Crawford DA, Jeffery RW, French SA. Television viewing, physical inactivity and obesity. Int J Obes 1999;23:437-40.

19. Cournot M, Ruidavets JB, Marquie JC, et al. Environmental factors associated with body mass index in a population of Southern France. Eur J Cardiovasc Prev Rehabil 2004;11:291-7.

20. Pulsford RM, Stamatakis E, Britton AR, et al. Sitting behavior and obesity: evidence from the Whitehall II study. Am J Prev Med 2013;44:132-8.

21. Katzmarzyk PT. Standing and mortality in a prospective cohort of Canadian adults. Med Sci Sports Exerc 2014;46:940-6. 